\title{
SÍNDROME DE KLEINE-LEVIN
}

RELATO DE UM CASO

OCTÁVIO DA SILVEIRA NETO * - OCTÁVIO AUGUSTO DA SILVEIRA **

RESUMO - Um caso de síndrome de Kleine-Levin é descrito em menino de 14 anos. O diagnóstico foi baseado nos sintomas de hipersonolência, megafagia e hipersexualidade, havendo boa resposta ao uso de cabarmazepina.

Kleine-Levin syndrome: a ease report.

SUMMARY - A case of a 14 year-old boy suffering from Kleine-Levin syndrome is described. The diagnosis was made by symptoms which suggested this disorder: megaphagia, hipersomnia and hipersexuality. A brief review is made emphasizing the pathophysiology, diagnosis and treatment of this syndrome which is frequently misdiagnosed.

Sonolência periódica com apetite mórbido foi descrita primeiramente por Kleine u em 1925, Lewis 16 em 1926, elaborada por Levin 15 em 1936 e denominada por Critchley e Hoftman* em 1942. A síndrome de Kleine-Levin é distúrbio neuropsiquiátrico caracterizado por hipersonolência, megafagia e alterações comportamentais: perda da inibição sexual, alucinações visuais e auditivas, ilusões. Acomete adolescentes de ambos os sexos, principalmente do sexo masculino e eventualmente adultos 1-7. Os ataques duram de 3 a 14 dias, havendo várias recorrências anuais. Entre as recorrências os pacientes apresentam-se normais, podendo lembrar-se de fatos ocorridos durante os ataques. A intensidade dos ataques diminui com o passar dos anos e pode haver, eventualmente, resolução espontânea.

Um caso da síndrome de Kleine-Levin é relatado.

\section{OBSERVAÇÃO}

RAV, paciente com 14 anos de idade, do sexo masculino, branco, católico, estudante, registro HC-UFPR 889193. Paciente sem passado de alterações psiquiátricas próprias ou de familiares, sendo internado no Hospital de Clínicas em 19/07/88. Há 6 meses apresentou cefaléia, febre, alteração de conduta e agitação psicomotora, sendo feito diagnóstico de meningite em outro serviço; há 19 dias apresentou odinofagia, cefaléia e febre, tendo sido diagnosticada infecção das vias aéreas superiores; há 14 dias apresentou sonolência e há 11 dias, distúrbio de conduta flutuante (sic); há 5 dias, em consulta médica ambulatorial foi diagnosticada amigdalite, sendo feito tratamento com antibióticos. Hoje, dia da internação, ao passear com o seu pai na rua, beijou compulsivamente três meninas. Ao exame físico apresentava-se afebril, sonolento e excessivamente confuso, masturbando-se em público e tomando outras atitudes indecorosas. Efetuada punção lombar em 19/07/88 em decúbito lateral, apresentando o líquido cefalorraqueano (LCR): pressão inicial $85 \mathrm{mmH20}$ e pressão final $50 \mathrm{mmH20}$; leucócitos 2/mm3, eritrócitos 3/mm3; proteínas $74 \mathrm{mg} \%$ glicose $61 \mathrm{mg} \%$. A tomografia computadorizada de crânio (TC) foi normal. Os electroencefalogramas (EEG), realizados em 21 e 22/ 07/88, em vigília e sonolência, mostraram lentifioação difusa com ausência de atividade irritativa. Apresentou durante o internamento bulimia, sendo feito o diagnóstico de síndrome de

Especialidade de Neurologia, Departamento de Clínica Médica, Hospital de Clínicas (HC), Universidade Federal do Paraná (UFPR): * Neurologista, Mestrando de Medicina Interna, HC, UFPR; ** Professor Adjunto, Chefe da Especialidade Neurologia, HC, UFPR

Dr. Octávio da Silveira Neto - Especialidade de Neurologia, Hospital de Clínicas, UFPR Rua General Carneiro 181 - 80069 Curitiba PR - Brasil. 
Kleine-Lievin. Iniciado tratamento com carbamazepina (600 mg/d.). Era 26/07/88 foi realizada novia punção lombar apresentando o LCR; aspecto límpico e incolor; leucócitos 2/mm3, eritrocitos 0/mm3; proteínas $69 \mathrm{mg} \%$; glicose $56 \mathrm{mg} \%$; imunologia para sífilis (VDRL) negativa; cultura para bactérias negativa; eletroforese de proteínas normal. Realizado novo EEG em 27/07/88, em vigília e sonolência espontânea, que se mostrou lento difuso, pouco modificado em relação aos anteriores. Após 4 dias do internamento houve diminuição da libido e o paciente apresentava-se menos confuso. Alta em 29/07/88, com melhora do quadro clínico. Reavaliado em consulta ambulatorial em 01/09/88, estava assintomático, frequentando a escola e nada lembrando do ocorrido por ocasião do seu internamento.

Reinternado em 17/11/88, apresentando sonolência excessiva e confusão há três dias. Tomando carbamazepina irregularmente há 30 dias. Ao exame apresentava-se sonolento e desorientado no tempo e espaço. Realizada punção lombar apresentando o LCR: pressão inicial 100 mmH20 e pressão final de $80 \mathrm{mmH20}$; aspecto límpido e incolor; leucócitos 4/mm3, eritrocitos 4/mm3; proteínas $78 \mathrm{mg} \%$; glicose $67 \mathrm{mg} \%$; EEG em 18/11/88, mostrou-se moderadamente lento difuso, com predomínio em regiões posteriores bilateralmente e, principalmente, em região occipital direita (delta polimórfico + ondas sharp); exame realizado em vigília e sono espontâneo. Em $21 / 11788$ persistia com sonolência, hiperfagia e aumento da libido. Realizada nova TC que foi novamente normal. Alta em 23/11/88 com melhora do quadro, sendo mantida a carbamazepina (600 mg/d.). Retornos ambulatoriais em 08/12/88, 13/01/89 e 22/02/89, apresentando-se assintomático e tendo vida social normal. Realizado novo EEG em 06/01/89 em vigília, hiperpnéia voluntária e sono espontâneo, sendo normal. O EEG em 28 / 02/89 mostrou-se lento difuso com sinais de atividade irritativa difusa; exame obtido em vigília, hiperpnéia voluntária [e sonolência espontânea. Nos retornos ambulatoriais de 10/04/ 89, 18/05/89, 20/07/89 e 11/10/89 apresentava-se assintomático. EEG realizado em 01/11/89 em vigília, hiperpnéia voluntária, foto-estimulação e sono induzido por hidrato de cloral: normal Apresentava-se, nesta ocasião, sem sintomas. Novo retorno ambulatorial em 11/04/90: assintomático.

\section{COMENTÁRIOS}

A etiologia da síndrome de Kleine-Levin ainda não está bem definida. Vários autores 10,11,13 evocam a possibilidade de uma lesão hipotalâmica ser a causa de tal síndrome. Estudos patológicos não são rotineiros, pois a doença tem evolução benigna. Porém, exames neuropatológicos em dois pacientes mostraram alterações no tálamo compatíveis a encefalite virai. Diversos casos têm como pródromo infecções virais e/ou encefalite. Gadoth e col.6, no teste de função pituitária-hipotalâmica «agudo», notaram resposta paradoxal do hormônio de crescimento (GH) à estimulação pelo hormônio tireotrófico (TRH), níveis plasmáticos basais de prolactina (PRL) boderline alto com resposta normal ao TRH; o padrão secretorio hormonal durante o sono mostrou anormalidades do hormônio luteinizante ( $\mathrm{LH}$ ) 11-hidroxi-corticosteróides (11-OHCS) e secreção de PRL em paciente masculino Ide 23 anos. Koerber e col.* acharam, em um paciente com síndrome de Kleine-Levin, níveis elevados de 5-hidroxitriptamina (5-TH) e 5-ácido hidroxiindolacético (5-HIAA) pós-mòrtem, sugerindo que a hipersonolência pode ser devida a diminuição da atividade noradrenérgica e a hiperatividade da via serotonérgica. Will e col. ${ }^{2}($ descreveram dois casos de síndrome de Kleine-Levin precipitados por traumatismo crânio-encefálico: um por acidente automobilístico e outro por luta de boxe. Striano e col. ${ }^{22}$ relataram um caso de Kleine-Levin associado a terror noturno. A polissonografia mostra tendência ao sono diurno, marcada por início precoce do período REM 14.16,25. Ocorre presença de dois ou mais períodos de início do sono em período REM. A latência do sono é geralmente menor que 5 minutos. O EEG mostra ondas delta difusas, com ou sem paroxismo 3. O diagnóstico diferencial deve ser feito com outras causas de hipersonolência, como: narcolepsia, apnéia do sono, uso de drogas e álcool, encefalopatías metabólicas, infecções e neoplasias do sistema nervoso central, doenças psiquiátricas (desordem de humor como na esquizofrenia, estados catatônicos ou depressão).

Estimulantes podem ocasionar alguma melhora na hipersonolência. Todavia, não corrigem outros comportamentos ou previnem novos ataques ${ }^{2} \wedge$ Antidepressivos tricíclicos e estimulantes centrais são úteis para a hipersonolência, contudo agravam outros sintomas comportamentais $\mathrm{u}-{ }^{2} ?$. O carbonato de lítio atua prevenindo alterações do humor, estabilizando o receptor dopaminérgico 8. Ogura e col. ${ }^{\wedge} \wedge$ utilizaram carbonato de lítio sem sucesso. Hart9 tratou com sucesso uma paciente de 15 anos com carbonato de lítio, sugerindo que a resposta ao tratamento implica em efeito sobre o metabolismo serotonérgico. Savet e col. ${ }^{21}$ utilizaram carbamazepina em um paciente de 19 anos; na época do relato do caso o paciente estava há 18 meses trabalhando normalmente, sem ter tido mais recorrências. 
O paciente cujo caso é aqui relatado apresenta todas as características clínicas para fazermos o diagnóstico da síndrome de Kleine-Levin: hipersonolência, megafagia, hipersexualidade e periodicidade de ataques, havendo boa resposta a terapêutica com carbamazepina. Os achados do LCR sugerem que os dois surtos podem ter sido precedidos por encefalite virai. Os demais exames laboratoriais foram normais exceto um EEG que mostrou sinais raros de atividade irritativa.

\section{REFERÊNCIAS}

1. Billiard M. The Kleine-Levin Syndrome. In Koella WP (ed): Sleep 1980, Proceedings of the 5th European Congress Sleep Research, Amsterdam: Basel: Karger, 1980, p 127.

2. Billiard M. The Kleine-Levin syndrome. In Kryger MH, Roth T, Dement WC (eds): Principles and Practice of Sleep Medicine. Baltimore: Saunders, 1989, p 377.

3. Critchley M. Periodic hypersomnia and megaphagia in adolescent males. Brain 1962, $85: 627$.

4. Critchley M, Hoffman HL. The syndrome of periodic somnolence and morbid hunger (Kleine-Levin syndrome). Br Med J 1942, 137.

5. Fergunson BG. Kleine-Levin syndrome: a case report. J Child Psychiat 1986, 27(2):275.

6. Gadoth N, Dyckerman Z, Bechar M, Laron Z, Lavie P. Episodic hormone secretion during sleep in Kleine-Levin syndrome: evidence for hypothalamic dysfunction. Brain Dev 1987, $9: 309$

7. Gerland H, Sumner D, Fourman P. The Kleine-Levin syndrome: some further observations. Neurology 1965. 15: 1161.

8. Goldberg MA. The treatment of Kleine-Levin syndrome with lithium. Can J Psychiat $1983,28: 491$.

9. Hart JH. Kleine-Levin syndrome: normal CSF monoamines and response of lithium therapy. Neurology 1985, 35:1395.

10. Jeffries JJ, Lefebvre A. Depression and mania associated with Kleine-Levin-Critchley syndrome. Can Psychiatr Assoc J 1973, $18: 439$.

11. Kleine W. Periodische Schlefsucht. Mschr Psychiatr Neurol 1925, 57 : 284

12. Koerber KR, Torkelson R, Haven G, Donaldson J, Cohen SM, Case M. Increased cerebrospinal fluid 5-hydroxytryptamine and 5-hydroxyindolacetic acid in Kleine-Levin syndrome. Neurology 1984, $34: 1597$.

13. Lauter H. Periodic diencephalic disorders: their relationship to maniac-depressive psychoses. Int J Neuropsych 1967, $3: 319$.

14. Lavie P, Gadoth N, Gordon CR, Goldhammer G, Bechar M. Sleep patterns in Kleine-Levin syndrome. Electroenceph Clin Neurophysiol 1979, $47: 369$

15. Levin M. Periodic somnolence and morbid hunger. Brain 1936, 59:494.

16. Lewis NDC. The psychoanalytic approach to the problem of children under twelve years of age. Psychoanal Rev 1926, $13: 424$.

17. Merriam AE. Kleine-Levin syndrome following acute viral encephalitis. Biol Psychiat 1986, $21: 1301$.

18. Messimy R, Weil B, Safar S. Sur un cas d'hypersomnie avec troubles des conduites alimentaires, excitation sexuelle et trouble du comportement. Sem Hop Paris 1967, 43:3100.

19. Ogura C, Okuma T, Nakazawa K, Kishimoto A. Treatment of periodic somnolence with lithium carbonate. Arch Neurol 1977, 27:143.

20. Reyndlds CF, Kupfer DJ, Christiansen CL, Auchenbach RC, Brenner RP, Sewitch DE, Taska LS, Coble PA. Multiple sleep latency test findings in Kleine-Levin syndrome. J Nerv Ment Dis 1984, 172:41.

21. Savet JF, Robert H, Angeli CH. Un cas de syndrome de Kleine-Levin stabilise depuis plus d'un an sous carbamazepine. Presse MM 1986, $15: 1281$.

22. Striano S, Bilo L, Meo R. An unusual case of Kleine-Levin syndrome associated with sleep terrors : Electroenceph Clin Neurophysiol 1986, 64 : 517.

23. Thompson C. Obrecht R, Franey C, Arendt J, Checkley SA. Neuroendocrine rhythms in a Kleine-Levin syndrome. $\mathrm{Br} \mathrm{J}$ Psychiat 1985, 147:440.

24. Waller DA, Jarriel S, Erman M, Emslie G. Recognizing and managing the adolescent with Kleine-Levin syndrome. J Adolesc Health Care 1984, 5: 139.

25. Wilkus RJ, Chiles JA. Electrophysiological changes during episodes of the Kleine-Levin syndrome. J Neurol Neurosurg Psychiat 1975, 38 : 1225.

26. Will RG, Young JP, Thomas DJ. Kleine-Levin syndrome: report of two cases with onset of symptoms precipitated by head trauma. Br J Psychiat 1988, 152 : 410.

27. Yassa R. Nair N. The Kleine-Levin syndrome: a variant? J Clin Psychiat 1978, 39 : 254. 